\title{
A FIXED POINT THEOREM FOR LOCALLY NONEXPANSIVE MAPPINGS IN NORMED SPACES
}

\author{
C. WATERS
}

\begin{abstract}
It is shown that global conditions in a recent result of W. A. Kirk can be replaced with the corresponding local conditions in case the domain is connected. Also a remark is made about the proof of the theorem referenced.
\end{abstract}

1. In this paper we adopt the notation of [2]. Let $X$ be a compact subset of a normed linear space $E$, and $T: X \rightarrow X$ be a mapping. We let $\Delta^{\prime} X$ denote the boundary of $X$ in $\overline{c o} X$. The mapping $T$ is locally nonexpansive (contractive) if for each $x \in X$ there exists $\varepsilon>0$ so that whenever $y$ and $z$ are distinct points in $X$ and $y, z \in B(x, \varepsilon),\|T(y)-T(z)\| \leqslant\|y-z\|(\|T(y)-T(z)\|<\|y-z\|)$. The mapping $T$ is called nonexpansive (contractive) if for each $x \in X, \varepsilon$ is unbounded.

A metric space $(X, d)$ is chainable if for each $\varepsilon>0$ and points $x$ and $y$ in $X$, there exists a finite set of distinct points $x=x_{1}, \ldots, x_{n}=y$ in $X$ so that $d\left(x_{i}, x_{i+1}\right)$ $\leqslant \varepsilon$ for each $i=1, \ldots, n-1$.

Rosenholtz [3] proved the following lemma.

LEMMA 1. Let $(X, d)$ be a compact and connected metric space. Then for each $\varepsilon>0$ and $x, y \in X$ there exists an $\varepsilon$-chain between $x$ and $y$, and the mapping $d_{\varepsilon}: X \times X \rightarrow R$, defined by

$$
d_{\varepsilon}(x, y)=\inf \left\{\sum_{i=1}^{n-1} d\left(x_{i}, x_{i+1}\right) \mid x=x_{1}, \ldots, x_{n}=y\right.
$$

$$
\text { is an } \varepsilon \text {-chain between } x \text { and } y\} \text {, }
$$

is a metric on $X$ equivalent to $d$. Furthermore, for each $x, y \in X$ and $\varepsilon>0$ there exists an $\varepsilon$-chain $x=x_{1}, \ldots, x_{n}=y$ so that

$$
d_{\varepsilon}(x, y)=\sum_{i=1}^{n-1} d\left(x_{i}, x_{i+1}\right) \text {. }
$$

2.

Lemma 2. Let $X$ be a compact connected subset of a Banach space. If $f: X \rightarrow X$ is locally nonexpansive on $X$ and locally contractive on $\Delta^{\prime} X$ there exists $\delta>0$ so that $f$ is

Received by the editors June 25, 1984 and, in revised form, July 15, 1985.

1980 Mathematics Subject Classification. Primary 47H10; Secondary 54H25.

Key words and phrases. Locally contractive, locally nonexpansive, chainable. 
nonexpansive and locally contractive on $\Delta^{\prime} X$ with respect to $d_{\delta}$ as defined in (1), and $d_{\delta}(x, y)=\|x-y\|$ if either $\|x-y\| \leqslant \delta$ or $[x, y] \subseteq X$.

Proof. By the compactness of $X$ there exists $\delta_{1}>0$ so that if $x, y \in X$ and $\|x-y\|<\delta_{1}$, then $\|f(x)-f(y)\| \leqslant\|x-y\|$. Also by the compactness of $\Delta^{\prime} X$ there exists $\delta_{2}>0$ so that for all distinct points $x, y \in \Delta^{\prime} X$ with $\|x-y\|<\delta_{2}, \| f(x)-$ $f(y)\|<\| x-y \|$. Let $\delta=2^{-1} \min \left\{\delta_{1}, \delta_{2}\right\}$. By Lemma 1 we may choose the metric $d \equiv d_{\delta}$ as defined in (1) to remetrize $X$. The second assertion in Lemma 2 easily follows from the definition of $d$ and the triangle inequality of the metric induced by the norm. To see the first assertion, let $x, y \in X$ and $x=x_{1}, \ldots, x_{n}=y$ be a $\delta$-chain in $X$ from $x$ to $y$ satisfying (2).

By the local nonexpansiveness of $f$

$$
\begin{aligned}
d(f(x), f(y)) & \leqslant \sum_{i=1}^{n-1} d\left(f\left(x_{i}\right), f\left(x_{i+1}\right)\right)=\sum_{i=1}^{n-1}\left\|f\left(x_{i}\right)-f\left(x_{i+1}\right)\right\| \\
& \leqslant \sum_{i=1}^{n-1}\left\|x_{i}-x_{i+1}\right\|=d(x, y) .
\end{aligned}
$$

Therefore $f$ is nonexpansive with respect to $d$.

The local contractiveness of $f$ with respect to $d$ follows from the definition of $\delta$, and the fact that $d(x, y)=\|x-y\|$ if $\|x-y\|<\delta$.

LEMMA 3. Let $X, f$, satisfy the hypotheses of Lemma 2, and d satisfy the conclusion of Lemma 2. If $x, y \in X, x=x_{1}, \ldots, x_{n}=y$ is a $\delta$-chain from $x$ to $y$ satisfying (2), and $d(f(x), f(y))=d(x, y)$, then there does not exist consecutive points of $x=$ $x_{1}, \ldots, x_{n}=y$ in $\Delta^{\prime} X$.

Proof. Suppose there exists $j \in\{1, \ldots, n-1\}$ so that $x_{j}, x_{j+1} \in \Delta^{\prime} X$. Then by Lemma 2 and (2),

$$
\begin{aligned}
d(f(x), f(y)) & \leqslant \sum_{i=1}^{n-1} d\left(f\left(x_{i}\right), f\left(x_{i+1}\right)\right) \\
& <\sum_{i=1}^{n-1} d\left(x_{i}, x_{i+1}\right)=d(x, y) .
\end{aligned}
$$

But (4) contradicts $d(f(x), f(y))=d(x, y)$.

THEOREM 1. Let $X$ be a compact nonempty connected subset of a Banach space. If $f$ : $X \rightarrow X$ is locally nonexpansive on $X$ and locally contractive on $\Delta^{\prime} X$, then $f$ has a fixed point.

Proof. Let $d$ be the metric as guaranteed in Lemma 2. For a subset $A$ of $X$, let $\bar{\delta}(A)$ and $\delta(A)$ be the $d$-diameter and norm diameter of $A$ respectively. By [2] we can choose a minimal invariant nonempty subset $M$ of $X$ with minimal $d$-diameter. By [1] $f$ restricted to $M$ is a $d$-isometry.

We first show $\delta(M)=\bar{\delta}(M)$. By Lemma 2 it is sufficient to show for all $m_{1}, m_{2} \in M,\left[m_{1}, m_{2}\right] \subseteq X$. For $m_{1}, m_{2} \in M$ let $m_{1}=x_{1}, \ldots, x_{n}=m_{2}$ be a $\delta$-chain satisfying (2). If for some $i=1, \ldots, n-1,\left[x_{i}, x_{i+1}\right] \nsubseteq X$, we may choose distinct 
points $z_{1}, z_{2} \in\left[x_{i}, x_{i+1}\right] \cap \Delta^{\prime} X$ so that $\left\|x_{i}-x_{i+1}\right\|=\left\|x_{i}-z_{1}\right\|+\left\|z_{1}-z_{2}\right\|+\| z_{2}$ $-x_{i+1} \|$. Then by (1)

$$
\begin{aligned}
d\left(f\left(m_{1}\right), f\left(m_{2}\right)\right) \leqslant & d\left(f\left(x_{1}\right), f\left(x_{2}\right)\right)+\cdots+d\left(f\left(x_{1}\right), f\left(z_{1}\right)\right) \\
& +d\left(f\left(z_{1}\right), f\left(z_{2}\right)\right)+\cdots+d\left(f\left(x_{n-1}\right), f\left(x_{n}\right)\right) \\
< & d\left(m_{1}, m_{2}\right) .
\end{aligned}
$$

But (5) contradicts that $f$ is a $d$-isometry on $M$. Hence for $i=1, \ldots, n-1$, $\left[x_{i}, x_{i+1}\right] \subseteq X$.

Let $\sigma \equiv d\left(m_{1}, m_{2}\right)$, and let $g:[0, \sigma] \rightarrow X$ be the arc length parametrization of the polygonal path

$$
P \equiv \bigcup_{i=1}^{n-1}\left[x_{i}, x_{i+1}\right]
$$

with $g(0)=x_{1}$ and $g(\sigma)=x_{n}$. Let

$$
A \equiv\left\{t \in[0, \sigma] \mid\left[m_{1}, g(s)\right] \subseteq X \text { for } s \in[0, t]\right\} .
$$

The set $A$ is trivially nonempty. Let $u \equiv \sup \{t \mid t \in A\}$. We show $u=\sigma$. Let $\left\{t_{n}\right\}$ be a sequence in $A$ with $\left\{t_{n}\right\} \uparrow u$, and $s \in[0,1]$. Then,

$$
\left\|(1-s) x_{1}+s g(u)-(1-s) x_{1}-s g\left(t_{n}\right)\right\|=s\left\|g(u)-g\left(t_{n}\right)\right\| .
$$

Since $X$ is closed and $g$ is continuous, (8) implies $(1-s) x_{1}+s g(u) \in X$. Hence $\left[x_{1}, g(u)\right] \subseteq X$.

If $u<\sigma$, we can choose a sequence $\left\{t_{n}\right\}$ in $[0, \sigma]$ decreasing to $u$, and points $z_{n}$, $y_{n}$ in $\left[m_{1}, g\left(t_{n}\right)\right]$ so that $\left\|z_{n}-y_{n}\right\|>\delta$ and $\left[z_{n}, y_{n}\right] \subset \operatorname{co} X \backslash X$. If for some $n y_{n}, z_{n}$ cannot be chosen so that $\left\|z_{n}-y_{n}\right\|>\delta$, we can form a $\delta$-chain $m_{1}=y_{1}, \ldots, y_{k}=$ $g\left(t_{n}\right)$ along $\left[m_{1}, g\left(t_{n}\right)\right]$ containing two consecutive boundary points so that

$$
\sum_{i=1}^{k-1}\left\|y_{i}-y_{i+1}\right\|=d\left(m_{1}, g\left(t_{n}\right)\right) \text {. }
$$

Then by (1) $m_{1}=y_{1}, \ldots, y_{k}, x_{j+1}, \ldots, x_{n}=m_{2}$, where $g\left(t_{n}\right) \in\left[x_{j}, x_{j+1}\right]$ is a $\delta$-chain satisfying (2) with respect to $m_{1}$ and $m_{2}$. Applying Lemma 3 we reach a contradiction. By the compactness of $\overline{c o} X$ we may assume there exist distinct points $y, z$ in $\overline{\operatorname{co}} X$ so that $z_{n} \rightarrow z$ and $y_{n} \rightarrow y$. By the continuity of $g$ and the definition of $\Delta^{\prime} X$

$$
[z, y] \subseteq\left[x_{1}, g(u)\right] \cap \Delta^{\prime} X .
$$

Let $g(u) \in\left[x_{j}, x_{j+1}\right]$. Then by (1)

$$
d\left(m_{1}, m_{2}\right)=\left\|m_{1}-g(u)\right\|+d\left(g(u), x_{j+1}\right)+\cdots+d\left(x_{n-1}, x_{n}\right) .
$$

By (9) we may choose a $\delta$-chain $m_{1}=y_{1}, \ldots, y_{k}=g(u)$ in $\left[m_{1}, g(u)\right]$ containing two consecutive points in $\Delta^{\prime} X$ satisfying $\left\|m_{1}-g(u)\right\|=\sum_{i=1}^{k-1} d\left(y_{i}, y_{i+1}\right)$. Then by

$$
\begin{aligned}
d\left(f\left(m_{1}\right), f\left(m_{2}\right)\right) \leqslant & d\left(f\left(y_{1}\right), f\left(y_{2}\right)\right)+\cdots+d\left(f\left(y_{k-1}\right), f\left(y_{k}\right)\right) \\
& +d\left(f\left(y_{k}\right), f\left(x_{j+1}\right)\right)+\cdots+d\left(f\left(x_{n-1}\right), f\left(x_{n}\right)\right) \\
< & d\left(m_{1}, m_{2}\right) .
\end{aligned}
$$


But (11) contradicts that $f$ restricted to $M$ is a $d$-isometry. Thus $\left[m_{1}, m_{2}\right] \subseteq X$. It now follows that $\delta(M)=\bar{\delta}(M)$.

Note that for each pair of points $m_{1}, m_{2}$ in $M$ with $d\left(m_{1}, m_{2}\right) \geqslant \delta$ there exists $\varepsilon>0$ so that if $y, z \in X$ with $y \in B_{d}\left(m_{1}, \varepsilon\right)$ and $z \in B_{d}\left(m_{2}, \varepsilon\right)$, then $d(y, z)=\| y$ $-z \|$. If this is not the case, there exist $m_{1}, m_{2} \in M$ with $d\left(m_{1}, m_{2}\right) \geqslant \delta$; so for each $\varepsilon>0$ there exist $y_{\varepsilon} \in B_{d}\left(m_{1}, \varepsilon\right), z_{\varepsilon} \in B_{d}\left(m_{2}, \varepsilon\right)$ and $a_{\varepsilon}, b_{\varepsilon} \in\left[y_{\varepsilon}, z_{\varepsilon}\right] \cap \operatorname{co} X \backslash X$ so that $\left\|a_{\varepsilon}-b_{\varepsilon}\right\|>\delta$. By the compactness of $\overline{c o} X$ we can choose distinct points $a, b$ in $X$ so that $[a, b] \subseteq\left[m_{1}, m_{2}\right] \cap \Delta^{\prime} X$. But then we can form a $\delta$-chain from $m_{1}$ to $m_{2}$ along $\left[m_{1}, m_{2}\right]$ containing consecutive points in $\Delta^{\prime} X$ and apply Lemma 3 to reach a contradiction.

Let $A \equiv\{(u, v) \in M \times M \mid d(u, v) \geqslant \delta\}$. By the compactness of $A$ we can choose a number $\varepsilon$ in $\left(0,3^{-1} \delta\right)$ so that if $(u, v)$ is in $A$ and $y, z \in X$ with $y \in B_{d}(u, \varepsilon)$ and $z \in B_{d}(v, \varepsilon)$, then $d(y, z)=\|y-z\|$. By the definition of $\varepsilon$ and by the triangle inequality,

$$
\text { for all } u, v \in M \text {, and } y \in B_{d}(u, \varepsilon) \text { and } z \in B_{d}(v, \varepsilon) \text {, }
$$

$$
d(y, z)=\|y-z\| \text {. }
$$

If $\delta(M) \neq 0$, we can choose distinct points $m_{1}, m_{2} \in M$ and $t \in(0,1)$ so that $x_{0} \equiv(1-t) m_{1}+t m_{2}$ is in the interior of $X$ with respect to co $X$ and $d\left(x_{0}, m_{1}\right)<\varepsilon$. Otherwise we can choose a $\delta$-chain satisfying (2) between $m_{1}$ and $m_{2}$ along $\left[m_{1}, m_{2}\right]$, containing two consecutive points in $\Delta^{\prime} X \cap B_{b}\left(m_{1}, \varepsilon\right)$ and contradict $d\left(f\left(m_{1}\right), f\left(m_{2}\right)\right)=d\left(m_{1}, m_{2}\right)$.

Let $N$ denote the set of nonnegative integers. Also for each $x \in X$, let $w(x)$ $\equiv \overline{\left\{f^{n}(x) \mid n \in N\right\}}$. By the minimality of $M, w\left(m_{1}\right)=M$ and for each $n \in N$, $f^{n}(M)=M$.

By the normal structure of $\operatorname{co} M$ there exist $y \in \operatorname{co} M$, and a real number $r$ satisfying $0<r<\delta(M)$ so that $\|y-m\| \leqslant r$ for all $m \in M$.

Since $x_{0}$ is in the interior of $X$ with respect to $\operatorname{co} X$ and $y \in \operatorname{co} X$, there exists $s \in(0,1)$ so that $z \equiv(1-s) x_{0}+s y \in B_{d}\left(m_{1}, \varepsilon\right) \cap X$. Then for all $m \in M$,

$$
\|z-m\| \leqslant(1-s)\left\|x_{0}-m\right\|+s\|y-m\| \leqslant(1-s) \delta(M)+s r .
$$

Let $\bar{r} \equiv(1-s) \delta(M)+s r$. Since $s \in(0,1), \bar{r}<\delta(M)$. Let $m, m_{n} \in M$ so that for all $n \in N, f^{n}\left(m_{n}\right)=m$. Then by the nonexpansiveness of $f$, and (12) and (13)

$$
\begin{aligned}
d\left(f^{n}(z), m\right) & =d\left(f^{n}(z), f^{n}\left(m_{n}\right)\right) \leqslant d\left(z, m_{n}\right) \\
& =\left\|z-m_{n}\right\| \leqslant \bar{r} .
\end{aligned}
$$

We show next that $\bar{\delta}(w(z))<\bar{\delta}(M)$, which will contradict the definition of $M$ and imply $\bar{\delta}(M)=0$.

By the continuity of $f$ it suffices to show for all $m, n \in N, d\left(f^{n}(z), f^{m}(z)\right) \leqslant \bar{r}$. Since $z \in \operatorname{co} M$, by (14), $\left\|f^{n}(z)-z\right\| \leqslant \bar{r}$. By (12) for $m, n \in N$ with $m>n$,

$$
d\left(f^{m}(z), f^{n}(z)\right) \leqslant d\left(f^{m-n}(z), z\right)=\left\|f^{m-n}(z)-z\right\| \leqslant \bar{r} .
$$

Therefore $\bar{\delta}(w(z)) \leqslant \bar{r}$. But (15) contradicts the definition of $M$. Hence $\bar{\delta}(M)=0$. Since $M \neq \varnothing, M=\{m\}$ for some $m \in X$. Therefore $f(m)=m$. 
3. We now comment on Theorem (1) in [2]. After choosing a minimal nonempty compact invariant set $M$ with minimal diameter, Kirk claimed $\operatorname{co} M \subseteq X$ by showing for all $m_{1}, m_{2} \in M,\left[m_{1}, m_{2}\right] \subseteq X$. Clearly it is not enough to show co $M \subseteq X$ unless one also shows $\left[m_{1}, m_{2}\right] \subseteq M$. However, one can avoid this situation by choosing an interior point $x_{0}$ as in Theorem 1 and then show $\delta\left(w\left(w_{0}\right)\right)<\delta(M)$.

\section{REFERENCES}

1. H. Freundenthal and W. Hurewicz, Duhnungen, Verkurzungen, Isometrien, Fund. Math. 26 (1936), $120-122$.

2. W. A. Kirk, Remarks on fixed points and boundaries, Proc. Amer. Math. Soc. 87 (1983), 62-64.

3. I. Rosenholtz, Evidence of a conspiracy among fixed point theorems, Proc. Amer. Math. Soc. 52 (1975), 312-318.

Department of Mathematics, Mankato State University, Mankato, Minnesota 56001 